\title{
INDUÇÃO DO PROCESSO DE REGENERAÇÃO DA VEGETAÇÃO DE CERRADO EM ÁREA DE PASTAGEM, ASSIS, SP
}

\author{
Giselda Durigan ${ }^{1}$ \\ Wilson A. Contieri ${ }^{1}$ \\ Geraldo A. D. C. Franco ${ }^{2}$ \\ Marco A. O. Garrido ${ }^{3}$
}

Recebido em 12/01/1999. Aceito em 21/06/1999

\begin{abstract}
RESUMO - (Indução do processo de regeneração da vegetação de cerrado em área de pastagem, Assis, SP). A vegetação natural do cerrado apresenta grande potencial de regeneração natural, principalmente através da brotação de raízes, ainda que se verifique a regeneração por sementes, em menor escala. Visando acelerar o processo de recuperação da cobertura arbustivo-arbórea em área de cerrado anteriormente utilizada como pastagem de Brachiaria decumbens em Assis, SP, foram testados seis tratamentos: A - Testemunha; B - Subsolagem; C - Preparo convencional de solo + calagem; D Aplicação de herbicida de amplo espectro; E - Herbicida seletivo para gramíneas; F - Preparo convencional do solo + herbicida pré-emergente. Os tratamentos foram aplicados após corte raso (roçada mecanizada) de toda a vegetação arbustivo-arbórea pré-existente na área experimental. A aplicação de herbicida de amplo espectro, controlando as gramíneas sem afetar as espécies do cerrado, proporcionou densidade $20 \%$ maior e cobertura $48 \%$ superior à testemunha, dois anos após a aplicação dos tratamentos. Nos tratamentos em que houve revolvimento do solo, os resultados foram inferiores à testemunha. $\mathrm{O}$ tratamento em que se fez também calagem, alterando a química do solo, apresentou os mais baixos valores de densidade e cobertura da vegetação de cerrado. Foram observadas 32 espécies do cerrado em regeneração, com predominância de Stryphnodendron obovatum, com 44,8\% dos indivíduos.
\end{abstract}

Palavras-chave - cerrado, regeneração natural, Brachiaria decumbens, manejo

\begin{abstract}
Inducement of cerrado regeneration in a pasture area, Assis, SP). Cerrado vegetation has a high potencial for natural regeneration. With the aim of accelerating the natural regeneration of cerrado woody species in an abandoned pasture of Brachiaria decumbens in Assis, São Paulo State, Brazil, six treatments were tested: A-control area; B- grubbing; C- tillage and liming; D- wide spectrum herbicide; E- selective herbicide for grasses, and F- tillage plus pre-emergence herbicide. Two years after the treatments, the application of a wide spectrum herbicide provided a density $20 \%$ higher and a crown cover $48 \%$ higher than in the control area. The treatments with intense soil manipulation a presented a density and a crown cover lower than in the control area. A total of 32 cerrado woody species were observed regenerating, $44,8 \%$ of individuals being from Stryphnodendron obovatum.
\end{abstract}

Key words - cerrado, natural regeneration, Brachiaria decumbens, management

1 Instituto Florestal, Estação Experimental de Assis, C. Postal 104, CEP 19800-000, Assis, SP, Brasil e-mail: giselda@femanet.com.br 


\section{Introdução}

As áreas originalmente cobertas por vegetação de cerrado lato sensu no Brasil correspondiam a $22 \%$ do território nacional (WWF \& PRÓCER 1995). Atualmente, apenas $1,47 \%$ da área original estão protegidos na forma de unidades de conservação de uso indireto, abaixo da média nacional que é de 2,61\% (Brasil 1998).

Além do tamanho reduzido das áreas legalmente protegidas, os remanescentes de cerrado, dispersos em pequenos fragmentos, têm sido alvo de distúrbios diversos que colocam em risco a estabilidade do ecossistema. Entre estes distúrbios, merecem destaque o aumento da frequuência de incêndios e a proliferação de gramíneas invasoras, que, além de afetar a diversidade biológica, têm o poder de intensificar sensivelmente os danos causados pelo fogo (Pivello 1992; WWF \& PRÓCER 1995; Baruch et al. 1996). A falta de conhecimento sobre como manejar estes fragmentos de cerrado tem levado a uma política de não manejá-los, nem mesmo nas unidades de conservação (Pivello 1992).

A pecuária nas regiões de cerrado, até pouco tempo atrás, era extensiva, baseada na vegetação nativa, de baixa produtividade. As gramíneas africanas, perfeitamente adaptadas às condições ambientais do cerrado, proporcionam aumento considerável de produtividade e, por isso, estão se tornando, segundo Klink (1994), as principais invasoras de áreas agrícolas, pastagens e mesmo de reservas naturais de cerrado.

Algumas destas gramíneas foram acidentalmente introduzidas no passado, como o capim-colonião (Panicum maximum Jacq.), o campim-jaraguá (Hyparrhenia rufa Nees Stanf.) e o capim-gordura (Melinis minutiflora Beauv.). No entanto, outras espécies têm sido intencionalmente introduzidas nos últimos anos, por proporcionar aumento de produtividade, principalmente a Brachiaria decumbens Stapf., seguida da Brachiaria umidicola (Rendel) Schwnickerdt e Andropogon gayanus Kunth.

Segundo Baruch et al. (1985), estas gramíneas exóticas são, geralmente, extremamente agressivas, capazes de invadir as áreas de cerrado e deslocar gramíneas nativas. A invasão, no entanto, parece ocorrer apenas em habitats perturbados (Bazzaz 1986; Klink 1996). Considerando-se que os remanescentes de cerrado estão sendo, na maioria das vezes, submetidos a distúrbios freqüentes, conclui-se que a ameaça de invasão é real em praticamente todos eles.

Nas áreas já invadidas, acidental ou intencionalmente, coloca-se a questão: como restaurar a vegetação de cerrado, restabelecendo-se a estrutura e os processos ecológicos do ecossistema original?

As espécies lenhosas do cerrado apresentam, geralmente, alto potencial de regeneração natural a partir de estruturas subterrâneas (Rizzini \& Heringer 1962; Goodland \& Ferri 1979; Hoffmann 1998). Embora existam evidências científicas de que a reprodução a partir de sementes é mecanismo importante para a regeneração de algumas espécies (Felipe \& Silva 1984; Franco et al. 1996a; 1996b; Oliveira \& Silva 1993), a regeneração por brotação tem maior êxito no processo de recuperação da cobertura vegetal do cerrado. Esta capacidade peculiar das espécies do cerrado, de recobrir o terreno a partir da rebrota de estruturas subterrâneas, depende das propriedades físicas e químicas do solo e do tempo decorrido após o desmatamento. 
Acredita-se que, através de interferências de manejo, seja possível acelerar o processo de regeneração e assegurar a conservação do ecossistema de cerrado. No entanto, segundo Pivello \& Coutinho (1996), informações que possam subsidiar ações de manejo são raras ou inexistentes, já que poucas pesquisas têm sido direcionadas para a solução de questões práticas. Na elaboração de modelos sucessionais que possam subsidiar o manejo dos cerrados, estes autores apresentam o uso combinado de fogo, pastoreio controlado e herbicidas como opções para o controle das espécies invasoras mais comuns no cerrado: Melinis minutiflora, Hyparrhenia rufa e Brachiaria spp.

O objetivo principal desta pesquisa é buscar tais subsídios, através da experimentação de diferentes técnicas de interferência no solo e sobre as espécies invasoras, que possam acelerar o processo de regeneração das espécies do cerrado.

\section{Material e métodos}

A Estação Experimental de Assis subordina-se ao Instituto Florestal e situa-se no município de Assis, SP ( $\left.35^{\prime} 22^{\circ} \mathrm{Se} 22^{\prime} 50^{\circ} \mathrm{W}\right)$, a altitude média de $500 \mathrm{~m}$. Predominam na área da Estação os solos do tipo Latossolo vermelho-escuro-álico, A moderado, textura média (LE1), que são solos ácidos e de baixa fertilidade, com elevados teores de alumínio. O clima, segundo a classificação de Köppen, é Cwa, ou seja, mesotérmico, com temperaturas dos meses mais frios inferiores a $18^{\circ} \mathrm{C}$, e dos meses mais quentes superiores a $22^{\circ} \mathrm{C}$. A precipitação anual gira em torno de $1.480 \mathrm{~mm}$ e ocorrem geadas esporádicas, tendo sido a mínima absoluta dos últimos 30 anos de $-2^{\circ} \mathrm{C}$ (SMA 1997). A vegetação original da Estação enquadra-se no conceito de cerrado lato sensu, predominando a fisionomia cerradão (Durigan et al. 1997).

A área experimental está inserida em uma área maior de $30 \mathrm{ha}$, incorporada à Estação Experimental de Assis um ano antes da instalação deste experimento. Durante os últimos 30 anos, esta área foi utilizada como pastagem, tendo sido, ao longo deste período, totalmente desmatada, submetida a roçadas periódicas e as gramíneas originais substituídas por Brachiaria decumbens.

A fisionomia da vegetação no local, antes da aplicação dos tratamentos, era de campo sujo, com indivíduos lenhosos de pequeno porte esparsos sobre a pastagem. Embora não tenha sido efetuado levantamento dessa vegetação, era evidente a predominância de Stryphnodendron obovatum (barbatimão).

Visando homogeneizar a cobertura da área experimental, toda essa vegetação lenhosa pré-existente foi eliminada através de roçada mecanizada. Deste modo, a cobertura do terreno por espécies arbustivo-arbóreas no momento da aplicação dos tratamentos era nula em todas as parcelas.

Considerando-se que a compactação do solo causada pelo pastoreio e a presença da Brachiaria spp. deveriam ser os principais obstáculos à regeneração da vegetação de cerrado, foram testados os seguintes tratamentos, visando superar estes obstáculos:
A. testemunha
B. subsolagem
C. preparo convencional de solo (aração e gradagem) + aplicação de calcário (3t/ha)


D. aplicação de herbicida de amplo espectro (Roundup, 4L/ha, cujo princípio ativo é o glifosato)

E. aplicação de herbicida seletivo para gramíneas (graminicida Post, 1,5L/ha, cujo princípio ativo é o Sethoxydim)

F. preparo convencional do solo + aplicação de herbicida pré-emergente (Surflan, $2 \mathrm{~L} / \mathrm{ha}$, cujo princípio ativo é a Orizalina).

Os tratamentos foram aplicados uma única vez, após corte raso de toda a vegetação arbustivo-arbórea da área experimental. Para cada tratamento foram instaladas quatro repetições, em blocos ao acaso, sendo a área de cada parcela de $720 \mathrm{~m}^{2}(60 \times 12 \mathrm{~m})$. A localização da área experimental pode ser visualizada na Fig. 1. As parcelas foram instaladas perpendicularmente a um fragmento de cerradão remanescente, tendo sido subdivididas, apenas para fins de análise de densidade da regeneração em função da distância do fragmento, em três sub-parcelas: 0 a $20 \mathrm{~m}, 20$ a $40 \mathrm{~m}$ e 40 a $60 \mathrm{~m}$.

Para avaliação da eficácia dos tratamentos, todos os indivíduos arbustivo-arbóreos presentes em cada parcela foram mapeados, identificados, numerados com etiquetas plásticas permanentes e medidos a altura e o diâmetro médio da copa. A partir destes dados, determinou-se a densidade (ind/ha) e a cobertura (\%) proporcionada pelas copas das espécies lenhosas do cerrado em regeneração.

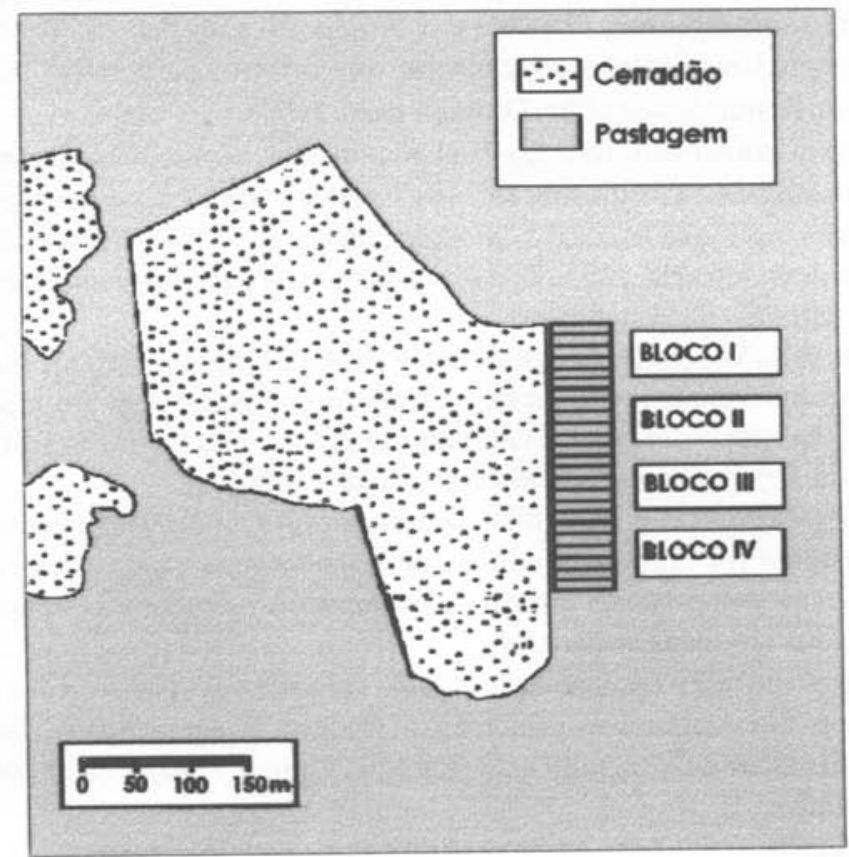

Figura 1. Localização da área experimental, com a posição das parcelas em relação ao fragmento de cerrado remanescente. 


\section{Resultados e discussão}

Os resultados obtidos em termos de densidade e cobertura da vegetação do cerrado em regeneração são apresentados nas Fig. 2 e 3.
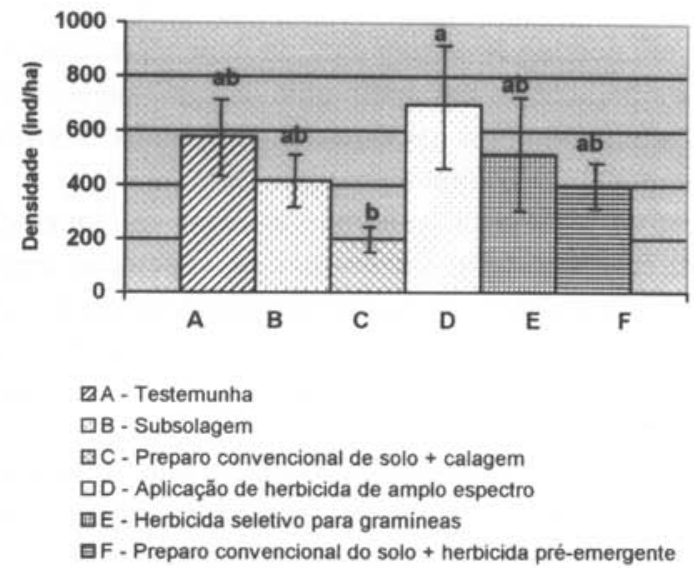

Figura 2. Densidade da vegetação arbustivo-arbórea do cerrado em área de pastagem de Brachiaria decumbens em Assis, SP, dois anos após a aplicação de diferentes tratamentos para indução da regeneração natural. Valores representados em colunas marcadas por letras distintas diferem entre si ao nível de significância de $5 \%$, pelo Teste de Tukey. As barras representam o desvio-padrão da média.

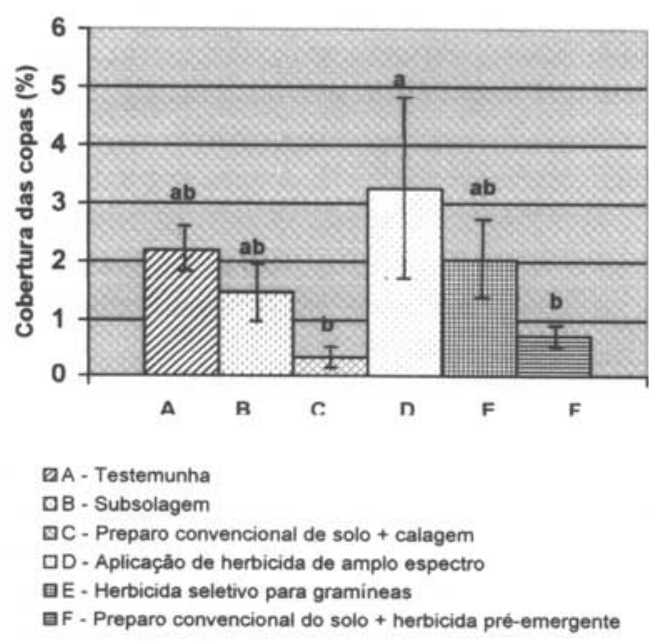

Figura 3. Cobertura da vegetação arbustivo-arbórea do cerrado em área de pastagem de Brachiaria decumbens em Assis, SP, dois anos após a aplicação de diferentes tratamentos para indução da regeneração natural. Valores representados em colunas marcadas por letras distintas diferem entre si ao nível de significância de $5 \%$, pelo Teste de Tukey. As barras representam o desvio-padrão da média. 
O melhor resultado foi obtido com a aplicação do herbicida de amplo espectro (ação total), que, controlando as gramíneas sem afetar as espécies do cerrado, resultou em densidade $20 \%$ maior e cobertura $48 \%$ superior à testemunha, dois anos após a aplicação dos tratamentos. A sobrevivência das espécies do cerrado ao herbicida devese, provavelmente, a algumas razões básicas: 1 . não afeta espécies lenhosas; 2 . por ser herbicida sistêmico, a eficácia do Roundup está na dependência de distribuição da fitomassa que favoreça a parte aérea em detrimento da parte subterrânea das plantas, ao contrário do que ocorre geralmente nas espécies do cerrado; 3. espécies de folhas coriáceas, comuns no cerrado, dificultam a absorção do produto.

O herbicida seletivo para gramíneas não exerceu qualquer efeito sobre a Brachiaria, resultando em densidade e cobertura da vegetação arbustivo-arbórea praticamente iguais às da testemunha. A recomendação técnica é de que este produto seja aplicado quando as gramíneas apresentam-se em fase inicial de desenvolvimento e, provavelmente por esta razão, não foi eficaz no controle da Brachiaria nas condições do experimento.

Todos os tratamentos em que houve revolvimento do solo apresentaram resultados inferiores à testemunha, especialmente aquele em que se fez também calagem, alterando a química do solo. Ao danificarem as estruturas subterrâneas remanescentes, estes métodos reduzem o potencial de regeneração por brotação.

Os solos de cerrado, de modo geral, são de forte a medianamente ácidos ( $\mathrm{pH}$ entre 4,0 e 5,5) e álicos, na maioria das vezes com saturação de alumínio superior a 50\% (Adámoli et al. 1985). Na Estação Experimental de Assis, em área próxima deste experimento, Durigan et al. (1987) verificaram pH entre 4,2 e 4,5, ou seja, solo fortemente ácido, com saturação de alumínio entre 43 e 50\%. Com a aplicação de três toneladas de calcário por hectare, certamente houve elevação de $\mathrm{pH}$ e redução da saturação de alumínio a níveis muito diferentes da situação anterior, fora dos limites mencionados para a ocorrência da vegetação de cerrado.

Alterações ambientais, entre elas a de pH, podem, segundo Pianka (1983), alterar os processos fisiológicos. Com a calagem, o solo possivelmente passou a ter características diferentes do ótimo fisiológico para os processos de regeneração das espécies pré-existentes no local, resultando em densidade e cobertura da vegetação lenhosa inferiores a todos os outros tratamentos.

A densidade de indivíduos arbustivo-arbóreos encontrada no melhor dos tratamentos, de 698 ind/ha aos dois anos, pode ser considerada promissora, já que plantios convencionais de revegetação utilizam por volta de 1.000 mudas/ha.

Encontraram-se 32 espécies arbustivo-arbóreas em regeneração (Tab. 1), com predominância absoluta de Stryphnodendron obovatum, com $44,8 \%$ dos indivíduos. A predominância desta espécie na área de estudo parece dever-se à sua predominância na área anteriormente à aplicação dos tratamentos e a uma capacidade de rebrota muito superior à das outras espécies em ambiente de pastagem, já que nos estudos fitossociológicos efetuados em cerrados da região (Durigan et al. 1987; 1997) esta espécie comporta-se como rara, com densidade relativa muito baixa e mesmo ausente em comunidades mais estáveis. 
Tabela 1 - Espécies arbustivo-arbóreas do cerrado em regeneração em área de pastagem de Brachiaria decumbens em Assis, SP, em ordem decrescente de densidade relativa.

\begin{tabular}{|c|c|c|}
\hline Espécie & Família & Densidade Relativa (\%) \\
\hline Stryphnodendron obovatum Benth & Mimosaceae & 44,8 \\
\hline Bredemeyera floribunda Willd & Polygalaceae & 8,4 \\
\hline Solanum paniculatum $\mathrm{L}$. & Solanaceae & $8,0^{*}$ \\
\hline Gochnatia barrosii Cabr. & Asteraceae & $6,9^{*}$ \\
\hline Myrcia bella Camb. & Myrtaceae & 5,8 \\
\hline Senna rugosa (G.Don) I. \& B. & Caesalpinaceae & 4,0 \\
\hline Baccharis dracunculifolia DC. & Asteraceae & $2,2 *$ \\
\hline Jacaranda caroba (Vell.) A. DC. & Bignoniaceae & 2,2 \\
\hline Aegiphila lhotzkyana Cham. & Verbenaceae & 2,1 \\
\hline Byrsonima intermedia A. Juss & Malpighiaceae & 2,0 \\
\hline Roupala montana Aubl. & Proteaceae & 2,0 \\
\hline Strychnos brasiliensis (Spreng.) Mart. & Loganiaceae & 1,8 \\
\hline Duguetia furfuracea (St. Hil.) Benth. \& Hook. & Annonaceae & 1,4 \\
\hline Machaerium acutifolium Vog. & Fabaceae & 1,1 \\
\hline Eugenia punicifolia (Kunth.) DC. & Myrtaceae & 0,9 \\
\hline Ocotea corymbosa (Musin.) Mez. & Lauraceae & 0,8 \\
\hline Annona coriacea Mart. & Annonaceae & 0,7 \\
\hline A. dioica Mart. & Annonaceae & 0,7 \\
\hline Copaifera langsdorffii Desf. & Caesalpinaceae & 0,7 \\
\hline Dimorphandra mollis Benth. & Caesalpinaceae & 0,6 \\
\hline Mabea fistulifera Mart. & Euphorbiaceae & 0,5 \\
\hline Tabebuia ochracea (Cham.) Standl. & Bignoniaceae & 0,5 \\
\hline Eugenia sp. & Myrtaceae & 0,3 \\
\hline Gochnatia polymorpha (Less.) Cabr. & Asteraceae & 0,2 \\
\hline Hymenaea stigonocarpa Mart. & Caesalpinaceae & 0,2 \\
\hline Machaerium brasiliense Vog. & Fabaceae & 0,2 \\
\hline Xylopia aromatica (Lam.) Mart. & Annonaceae & 0,2 \\
\hline Annona cacans Warm. & Annonaceae & 0,1 \\
\hline Pera glabrata (Schott.) Baill. & Euphorbiaceae & 0,1 \\
\hline Qualea multiflora Mart. & Vochysiaceae & 0,1 \\
\hline Terminalia brasiliensis Camb. & Combretaceae & 0,1 \\
\hline Tocoyena formosa (Cham.\& Schlecht) K. Schum. & Rubiaceae & 0,1 \\
\hline
\end{tabular}

* Espécies das quais foram observados indivíduos provavelmente oriundos de sementes.

Ainda que o diagnóstico preciso do modo de regeneração não tenha sido possível para todas as plantas, o porte dos indivíduos, a agregação das populações de algumas espécies e a presença de estruturas subterrâneas preexistentes nos indivíduos em regeneração são indícios que levam à estimativa de que pelo menos $80 \%$ das plantas encontradas na área experimental são oriundas de rebrota. Dentre as espécies que apresentaram indivíduos provavelmente oriundos de sementes destacam-se: Solanum paniculatum, Gochnatia barrosii e Baccharis dracunculifolia.

Os dados de densidade das plantas em diferentes distâncias do fragmento de cerrado remanescente não apontaram diferenças significativas um ano após a aplicação dos tratamentos, indicando que a proximidade do fragmento não afeta a densidade dos indivíduos em regeneração. Foram encontrados, em média, 342 indivíduos por hectare entre 0 e $20 \mathrm{~m}$ de distância do fragmento, 387 ind./ha entre 20 e $40 \mathrm{~m}$ e 351 ind./ha entre 40 e $60 \mathrm{~m}$. Esta inexistência de relação entre a densidade das plantas e a distância do 
fragmento leva a crer que a chuva de sementes não seja mecanismo indutor importante da regeneração natural para a vegetação de cerrado.

Dentre todos os tratamentos testados nas condições da área de estudo, o controle da Brachiaria, através da aplicação de herbicida, mostrou-se como o único tratamento capaz de acelerar significativamente a regeneração da vegetação do cerrado. No entanto, a utilização de herbicidas como técnica de manejo visando a conservação de ecossistemas naturais é vista com restrições por alguns setores da comunidade científica e pelos movimentos ambientalistas de modo geral.

Herbicidas são, por definição, substâncias destinadas a controlar a vegetação, e não a favorecer o seu desenvolvimento. Estão incluídos entre os chamados agrotóxicos e a eles são atribuídos graves efeitos impactantes sobre os ecossistemas. Essa generalização, no entanto, é equivocada. Há muitos herbicidas diferentes no mercado, cada um com especificações e efeitos distintos. Pelo seu modo de ação, o herbicida de ação total utilizado (Roundup), que favoreceu a regeneração das plantas de cerrado, é praticamente imóvel nos solos (Mensik \& Janssen 1994), não sofrendo lixiviação e reduzindo os riscos de contaminação dos recursos hídricos. Além disso, o tempo relativamente curto de permanência no sistema reduz consideravelmente a possibilidade de outros impactos ambientais negativos. A decomposição do glifosato ocorre essencialmente por degradação microbiana, sem que haja qualquer efeito negativo sobre os microrganismos (Sprankle et al. 1975).

Outras alternativas de manejo que visem a redução da competição das gramíneas invasoras com as espécies nativas devem ser testadas, como, por exemplo, o pastoreio controlado, já mencionado por Pivello \& Coutinho (1996), podendo trazer resultados favoráveis e menor rejeição nos meios conservacionistas.

\section{Referências bibliográficas}

Adámoli, J.; Macêdo, J.; Azevedo, L. G. \& Madeira Netto, J. 1985. Caracterização da região dos cerrados Pp. 33-74 In W. J. Goedert (ed.). Solos dos cerrados: tecnologias e estratégias de manejo. Nobel, São Paulo e EMBRAPA/CPAC, Brasília.

Baruch, Z.; Belsky, A. J.; Bulla, L.; Franco, A.; Garay, I.; Haridasan, M.; Lavelle, P.; Medina, E. \& Sarmiento, G. 1996. Biodiversity as regulator of energy flow, water use and nutrient cycling in savannas Pp. 175194. In: O. T. Solbrig; E. Medina \& J. F. Silva (Eds.), Biodiversity and Savanna Ecosystem Processes. (Ecological Studies, 121). Springer-Verlag Berlin, Heidelberg.

Baruch, Z.; Ludlow, M. M. \& Davis, R. 1985. Photosynthetic responses of native and introduced $C_{4}$ grasses from Venezuelan savannas. Oecologia 67: 388-393.

Bazzaz, F.A. 1986. Life history of colonizing plants: some demographic, genetic and physiological features Pp. 96-110. In H. Mooney \& M. Drake (Eds.), Ecology of biological invasions of North America and Hawaii. Springer-Verlag, New York.

Brasil. 1998. Primeiro relatório nacional para a Convenção sobre Diversidade Biológica: Brasil. Ministério do Meio Ambiente, dos Recursos Hídricos e da Amazônica Legal, Brasília.

Durigan, G.; Saraiva, I. R.; Garrido, M. A. O.; Gurgel-Garrido, L. M. A. \& Peche Filho, A. 1987. Fitossociologia e evolução da densidade da vegetação do cerrado em Assis, SP. Boletim Técnico IF 41(2): 59-78.

Durigan, G.; Franco, G. A. D. C.; Pastore, J. A. \& Aguiar, O. T. 1997. Regeneração natural da vegetação de cerrado sob floresta de Eucalyptus citriodora. Revista do Instituto Florestal 9(1): 71-85.

Felippe, G. M. \& Silva, J. C. S. 1984. Estudos de germinação em espécies do cerrado. Revista Brasileira de Botânica 7: 157-163. 
Franco, A. C.; Nardoto, G. B. Souza, M. P. 1996a. Patterns of soil water potential and seedling survival in the cerrados of Central Brazil Pp. 277-280. In Anais do IX Simpósio Sobre o Cerrado. 1996. EMBRAPA/CPAC, Brasília.

Franco, A. C.; Nardoto, G. B. Souza, M. P. 1996b. Estabelecimento e crescimento de Dalbergia miscolobium Benth. em áreas de campo sujo e cerrado no DF Pp. 84-92. In H. S. Miranda; C. H. Saito \& B. F. S. Dias (Orgs.), Impactos de queimadas em áreas de cerrado e restinga. UnB, ECL, Brasília.

Goodland, R. \& Ferri, M. G. 1979. Ecologia do Cerrado. Ed. Itatiaia e EDUSP, São Paulo.

Hoffmann, W. A. 1998. Post-burn reproduction of woody plants in a neotropical savanna: the relative importance of sexual and vegetative reproduction. Journal of Applied Ecology 35: 422-433.

Klink, C. A. 1994. Effects of clipping on size and tillering of native and African grasses of the Brazilian savannas (the cerrado). Oikos 70: 365-376.

Klink, C. A. 1996. Germination and seedling establishment of two native and one invading African grass species in the Brazilian cerrado. Journal of Tropical Ecology 12: 139-147.

Mensink, H. \& Janssen, P. 1994. Glyphosate. Environmental Health Criteria 159. World Health Organization, Geneva.

Oliveira, P. E. \& Silva, J. C. S. 1993. Reproductive biology of two species of Kielmeyera (Guttiferae) in the cerrados of Central Brazil. Journal of Tropical Ecology 9: 67-79.

Pianka, E. R. 1983. Evolutionary Ecology. 3 ed. Harper \& Row Publishers, Inc., New York.

Pivello, V. R. 1992. An expert system for the use of prescribed fire in the management of Brazilian savannas. Ph. D Thesis. Imperial College, University of London, London

Pivello, V. R. \& Coutinho, L. M. 1996. A qualitative successional model to assist in the management of Brazilian cerrados. Forest Ecology and Management 87: 127-138.

Rizzini, C. T. \& Heringer, E. P. 1962. Studies on the underground organs of the trees and shrubs from some southern Brazilian savannas. Anais da Academia Brasileira de Ciências 34: 235-247.

SMA. 1997. Estação Experimental e Ecológica de Assis. Secretaria do Meio Ambiente do Estado de São Paulo, São Paulo.

Sprankle, P.; Meggit, W. F. \& Penner, D. 1975. Adsorption, mobility and microbial degradation of glyphosate by soils. Weed Science 23(3): 229-234.

WWF \& PRÓCER, 1995. De grão em grão o cerrado perde espaço. PRÓ-CER/WWF \& PRÓCER, Brasília. 